\title{
Use of a modified glass-based dish for long-term observations of Coleochaete culture without dew condensation on the lid
}

\author{
Daisuke Tamaoki $^{1,2,3}$, Aki Fukuda ${ }^{1}$, Hisato Ikegaya ${ }^{1}$, Tomonori Nakai ${ }^{1}$, Daisuke Yamauchi ${ }^{1}$, Yoshinobu Mineyuki ${ }^{1,2}$ \\ ${ }^{1}$ Graduate School of Life Science, University of Hyogo, Shosha 2167, Himeji, Hyogo, 671-2280 Japan \\ ${ }^{2}$ The Japan Science and Technology Agency (JST), SENTAN \\ ${ }^{3}$ Present Address: Division of Functional Genomics, Advanced Science Research Centre, Kanazawa University, Kanazawa, Takara- \\ machi 13-1, Kanazawa, Ishikawa, 920-0934 Japan \\ Author for correspondence: Y. Mineyuki, mineyuki@sci.u-hyogo.ac.jp
}

Summary: Insertion of a sheet of cover glass in the lid of a glass-based dish is a useful technique for observation by differential interference contrast microscopy. However, microscopic observations of algal cultures using this glass-based dish are often interfered with by dew condensation on the glass surface of the lid when the culture is incubated for a long time. To prevent dew development on the lid surface during incubation, we employed a glass-based dish whose inner surface of the glass-lid is coated with 2-methacryloyloxyethyl phosphorylcholine polymer. Using this modified dish, we could observe thalli development of Coleochaete scutata successively for four weeks without dew condensation on the lid.

Key words: culture dish, MPC polymer, Coleochaete, long-term DIC observation, glass-based dish

\section{INTRODUCTION}

Coleochaete scutata forms a flat and radial thallus as a result of repeated anticlinal and periclinal divisions of the outermost cell layer (Marchant and Pickett-Heaps 1973). Because of its morphological simplicity that makes every cell observable and an ease of culture, C. scutata is considered a model system for plant morphogenesis (Dupuy et al. 2010). To understand how cell growth and division contribute to the body shaping of $C$. scutata, long-term observation of the developmental processes of the multicellular thallus from a single cell is necessary.

Observation of algal culture in a glass-based dish by differential interference contrast (DIC) microscopy is a useful technique for this purpose. To achieve a high-precision observation by DIC microscopy, it is important to avoid light path interference by plastic materials. For this purpose, a glassbased dish with a sheet of cover glass inserted into the lid has been previously used, and some types of these dishes are now commercially available (e.g., SkyLight Glass Based Dish, AGC Techno Glass Co. Ltd., Shizuoka, Japan and Glass bottom dish D111303, Matsunami Glass Ind., Ltd., Kishiwada, Japan). However, dew condensation on the inner surface of glass-lids occurs during the long-term observation of algal culture and reduces the image quality of DIC observation.

2-Methacryloyloxyethyl phosphorylcholine (MPC) is a methacrylate monomer with a phosphorylcholine group, and the polymer of MPC has wide clinical applications (Iwasaki and Ishihara 2012). As MPC-polymer coating can provide high hydrophilicity to the material surface (Iwasaki and Ishihara 2012), this polymer coating on the glass surface has the potential to prevent the occurrence of dew condensation on the glass surface. In this study, we tested the applicability of modified glass-based dish equipped with a lid with an MPC polymercoated cover glass on its inner surface to observe algal culture for long-term.

\section{MATERIALS AND METHODS}

\section{Culture of Coleochaete scutata}

C. scutata (CPCC305) was obtained from Canadian Phycological Culture Centre. Axenic culture was preserved on $3 \mathrm{~N}+$ medium (Starr 1978), modified as described below, and Closterium medium (Ichimura 1971), with minor modifications as described below, under a 16-h light (fluorescent lamps) and 8-h dark regime at $20^{\circ} \mathrm{C}$. The light intensity was about $20 \mu \mathrm{mol}$ photons $\mathrm{m}^{-2} \mathrm{~s}^{-1}$. One liter of $3 \mathrm{~N}+$ medium contained $750 \mathrm{mg} \mathrm{NaNO}$, $25 \mathrm{mg} \mathrm{CaCl} 2 \cdot 4 \mathrm{H}_{2} \mathrm{O}, 75 \mathrm{mg} \mathrm{MgSO}_{4} \cdot 7 \mathrm{H}_{2} \mathrm{O}, 75 \mathrm{mg} \mathrm{K} \mathrm{NHPO}_{4}$, $750 \mathrm{mg} \mathrm{NaNO}$, $25 \mathrm{mg} \mathrm{NaCl}, 175 \mathrm{mg} \mathrm{KH}_{2} \mathrm{PO}_{4}, 3 \mathrm{ml}$ PII trace metals $\left(1.14 \mathrm{~g} \mathrm{~L}^{-1} \mathrm{H}_{3} \mathrm{BO}_{3}, 49 \mathrm{mg} \mathrm{L}^{-1} \mathrm{FeCl}_{3} \cdot 6 \mathrm{H}_{2} \mathrm{O}, 123 \mathrm{mg} \mathrm{L}^{-1}\right.$ $\mathrm{MnSO}_{4} \cdot \mathrm{H}_{2} \mathrm{O}, 22 \mathrm{mg} \mathrm{L}^{-1} \mathrm{ZnSO}_{4} \cdot 7 \mathrm{H}_{2} \mathrm{O}, 5 \mathrm{mg} \mathrm{L}^{-1} \mathrm{CoSO}_{4} \cdot 7 \mathrm{H}_{2} \mathrm{O}$, $1 \mathrm{~g} \mathrm{~L}^{-1}$ disodium ethylenediaminetetraacetic acid), and soil extract. To prepare the soil extract, $56.7 \mathrm{~g}$ of soil purchased from a garden store was autoclaved in $0.75 \mathrm{~g} \mathrm{~L}^{-1} \mathrm{CaCO}_{3}$ for $2 \mathrm{~h}$ at $121^{\circ} \mathrm{C}$, and the suspension was filtered through a piece of gauze. One liter of Closterium medium contained $150 \mathrm{mg}$ $\mathrm{Ca}\left(\mathrm{NO}_{3}\right)_{2} \cdot 4 \mathrm{H}_{2} \mathrm{O}, 100 \mathrm{mg} \mathrm{KNO} 3,40 \mathrm{mg} \mathrm{MgSO} \cdot 7 \mathrm{H}_{2} \mathrm{O}, 50 \mathrm{mg}$ disodium $\beta$-glycerophosphate $5 \mathrm{H}_{2} \mathrm{O}, 0.1 \mu \mathrm{g}$ vitamin $\mathrm{B}_{12}, 0.1$ $\mu \mathrm{g}$ biotin, $10 \mu \mathrm{g}$ thiamine $\mathrm{HCl}, 500 \mathrm{mg}$ Tris (hydroxymethyl) aminomethane and $3 \mathrm{~mL}$ PIV metal solution, with $\mathrm{pH}$ adjusted to 7.5 using $\mathrm{KOH}$. The PIV metal solution contained $750 \mathrm{mg}$ disodium ethylenediaminetetraacetic acid, $194 \mathrm{mg} \mathrm{FeCl} 3 \cdot 6 \mathrm{H}_{2} \mathrm{O}$, $82 \mathrm{mg} \mathrm{MnCl}_{2} \cdot 4 \mathrm{H}_{2} \mathrm{O}, 10 \mathrm{mg} \mathrm{ZnCl} 2,4 \mathrm{mg} \mathrm{CoCl}{ }_{2} \cdot 6 \mathrm{H}_{2} \mathrm{O}$ and 8 mg Na $2 \mathrm{MoO}_{4} \cdot 2 \mathrm{H}_{2} \mathrm{O}$ per liter. Two mililiters of the medium and a small aliquot of culture medium containing C. scutata were added to a culture dish of $35-\mathrm{mm}$ diameter.

\section{Glass-based dish}

For the control experiment, two types of 35-mm-diameter glass-based dish with a glass-lid, SkyLight Glass Based Dishes (3970-035-SK, AGC Techno Glass Co. Ltd.) and Glass bottom dish D111303 (Matsunami Glass Ind., Ltd.) were used. The bottom of SkyLight Glass Based Dishes has a round cover 
glass of 27-mm diameter with a lid that has a round cover glass of 30-mm diameter. To prepare a culture dish for a long-term observation, the inner surface of the glass-lids of SkyLight Glass Based Dishes was coated with MPC polymer (custom-made, 3970-035-SK-DEF, Lot 121101-35GD; AGC Techno Glass Co. Ltd.). This method of MPC-polymer coating (Ishihara et al. 2011) has been used in some cell culture products from AGC Techno Glass Co. Ltd., such as low-adhesion surface dishes and well plates (EZ-BindShut II Series) and cell culture ware for spheroid formation (EZSPHERE dishes).

\section{Image acquisition}

DIC images of thalli were acquired using an inverted microscope (ECLIPSE Ti, Nikon Co., Tokyo, Japan) equipped with 10x (NA 0.30 ) or a $20 \times(\mathrm{NA} 0.75$ ) objective lenses, and an EM-CCD camera (iXon DV897-BV, Andor Technology PLC, Belfast, UK) in the GLIM system (Tamaoki and Mineyuki 2012). Images of the culture dish were acquired by using a digital camera (Digital Sight DS-5M, Nikon Co.) equipped with a macro lens (OMSystem Zuiko Auto-Macro 50 mm F3.5, Olympus Co., Tokyo, Japan).

\section{RESULTS AND DISCUSSION}

Thalli of C. scutata grown at $20^{\circ} \mathrm{C}$ in a SkyLight Glass Based Dish or in a Glass bottom dish D111303 were observed. In both cases, dew condensation occurred within 7 days (Figure 1A), and the dew on the glass-lid obstructed a view (Figure 1B). Then, we used SkyLight Glass Based Dishes and compared results obtained using dishes with and without MPC-polymer coating on the inner surface of the lids. C. scutata culturing was initiated after adding a small amount of $C$. scutata to a dish that contained $2 \mathrm{~mL}$ fresh medium. Dishes were incubated at $20^{\circ} \mathrm{C}$ during the culture. In dishes without the MPC-polymer coating, dew condensation began to occur on the glass surface of the lid a few days after C. scutata inoculation. Dew condensation expanded to the whole surface at 28 days after culture (Figure 2A). In contrast, dew condensation did not occur on the glass surface of the lid of the dish with MPC polymer-coated glass (Figure 2B) and the thalli of C. scutata were clearly observed by DIC microscopy (Figure 2C). Because MPC polymer coated only the glass part of the lid, dew condensation still occurred on the plastic rim (a non-coated region) of the lid (Figure 2B). This result showed that coating a glass-lid with MPC-polymer prevented the dew condensation on the glass surface during culture at $20^{\circ} \mathrm{C}$ for 28 days.

After inoculating C. scutata into the fresh liquid medium in the MPC polymer-coated SkyLight Glass Based Dishes, we observed the development of $C$. scutata thallus successively by DIC microscopy for 28 days. Thallus development of $C$. scutata from a single cell was observed (Figure 3). Single cells
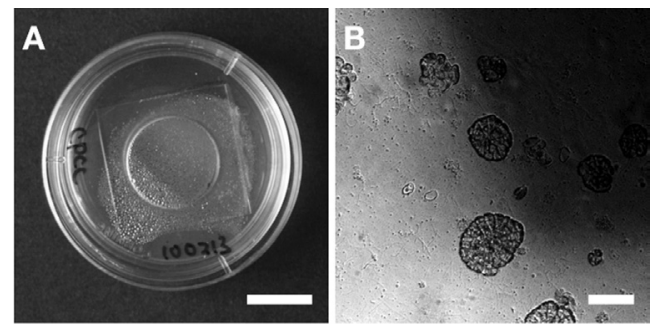

Figure 1 Dew condensation on the inner surface of a glass-lid reduced image quality of DIC observation. (A) An image of a lid of culture dish (Glass bottom dish D111303) kept for 6 days at $20{ }^{\circ} \mathrm{C}$. (B) A DIC image of C. scutata thalli with dew condensation on the glass-lid. Thalli of $C$. scutata were cultured for 31 days. Bars $=1$ $\mathrm{cm}(\mathrm{A})$ and $=100 \mu \mathrm{m}(\mathrm{B})$.

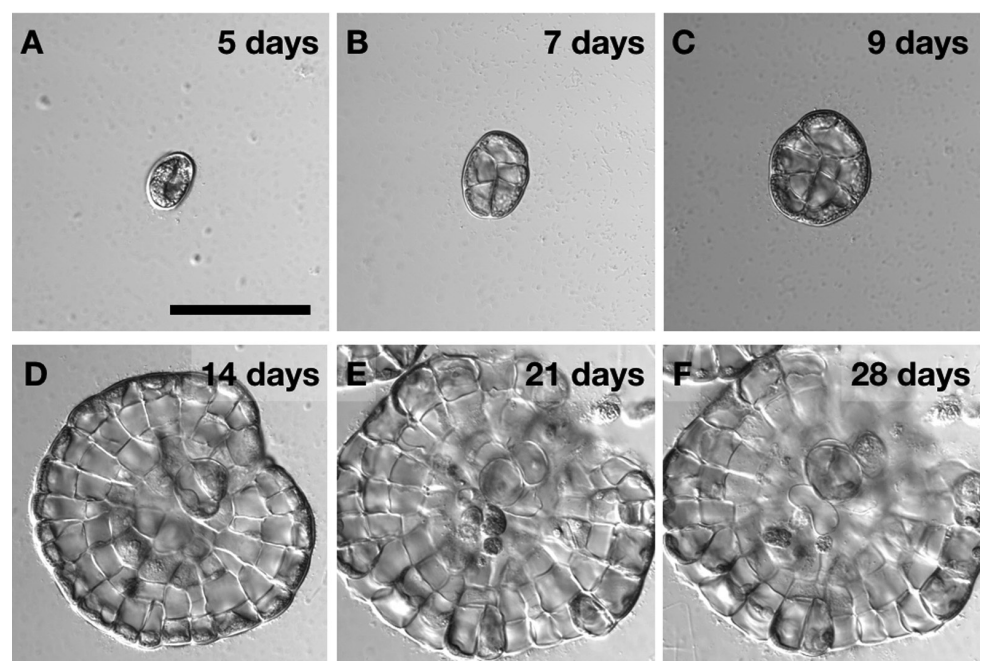

Figure 3 A series of DIC images of $C$. scutata thallus in the dish with MPC-polymer coating. The thallus was observed by DIC microscopy for 28 days. Images (A) to (F) were taken $5,7,9,14,21$, and 28 days after inoculation, respectively. Bar $=50 \mu \mathrm{m}$. 
of C. scutata were attached to the surface of the cover glass at the bottom of the dish at a few days after inoculation (Figure $3 \mathrm{~A})$. These cells began to divide and started to form a thallus 7 days after inoculation (Figure 3B). The thallus of C. scutata became disc-shaped (Figure 3C) and grew by repeated cell division of the marginal cell layers for the next 14 days (Figures 3D and E). Zoospore formation started about 14 days after inoculation (Figure 3D). The growth rate of the thallus gradually then decreased, and the thallus growth ceased 21 days after inoculation (Figures $3 \mathrm{E}$ and $\mathrm{F}$ ). Without dew condensation on the inner surface of the lid, we could easily observe the thallus development from a single cell for 28 days (Figure 3).

Present study clearly showed that this MPC-polymer coating helps prevention of dew condensation and provides a good optical condition for long-term DIC observation of thalli development of C. scutata. The long-term observation is a useful method to elucidate the mechanisms of pattern formation (Dupuy et al. 2010), reproduction (Ichimura 1971) and colony formation of algae. The 35-mm-diameter glass-bottom dish with a glass-lid coated with MPC-polymer presented in this paper will be a useful dish for such long-term observations of various types of algal and plant tissue cultures in a liquid medium. Various types of culture vessels are used for plant and algal studies. In a case when the obstruction of a view occurs by dew condensation in these culture vessels, the MPC-polymer coating on the inner surface of these culture vessels may be a useful for observation of plants or algae.

\section{ACKNOWLEDGEMENT}

We are grateful to Canadian Phycological Culture Centre for kindly supplying us with Coleochaete scutata for the present study. This work was partly supported by the Japan Science and Technology Agency (JST). We also thank Mr. Hideo Genkaku (AGC Techno Glass Co. Ltd.) for useful advices on MPCpolymer coated dishes. We would like to thank Editage (www. editage.jp) for English language editing.

\section{REFERENCES}

Dupuy, L., Mackenzie, J., and Haseloff, J. (2010) Coordination of plant cell division and expansion in a simple morphogenetic system. Proc Natl Acad Sci USA 107: 2711-2716.

Ichimura, T. (1971) Sexual cell division and conjugation-papilla formation in sexual reproduction of Closterium strigosum. In: Nishizawa K. (ed), Proceedings of the 7th International Seaweed Symposium, University of Tokyo Press, Tokyo, pp. 208-214.

Ishihara, K., Takai, M., and Nishizawa, K. (2011) University of Tokyo, Japanese Patent P4729709, 2011-04-28.

Iwasaki, Y. and Ishihara, K. (2012) Cell membrane-inspired phospholipid polymers for developing medical devices with excellent biointerfaces. Sci Technol Adv Mater 13: 064101.

Marchant, H. V. and Pickett-Heaps, J. D. (1973) Mitosis and cytokinesis in Coleochete scutata. J Phycol 9: 461-471.

Starr, C. R. (1978) The culture collection of algae at the University of Texas at Austin. J Phycol 14: Suppl 47-100.

Tamaoki, D. and Mineyuki, Y. (2012) A microscope system for recording the local structural dynamics and the whole cell events in parallel. Plant Morphol 24: 13-17.

Received: 27 February 2016 /Accepted: 24 March 2016 